\title{
Identifying coeliac disease in DXA referrals
}

\author{
M. W. J. Davie
}

Received: 21 May 2014 / Accepted: 6 June 2014 / Published online: 28 June 2014

(C) International Osteoporosis Foundation and National Osteoporosis Foundation 2014

Dear Editor,

Hjelle and colleagues [1] draw attention to the association of coeliac disease $[\mathrm{CD}]$ with fracture and discuss whether patients presenting with idiopathic osteoporosis should have serological testing for $\mathrm{CD}$. Apart from the complications affecting the bone, they also draw attention to the possibility of increased morbidity and mortality in CD.

In a service-based evaluation of serological testing for coeliac disease, women over 50 years, without a diagnosis of gluten sensitivity, referred for DXA scans underwent serological testing for coeliac disease if they fulfilled the following conditions: history of fracture or a BMI $<21 \mathrm{~kg} / \mathrm{m}^{2}$ (1 SD below the mean for referrals) plus a BMD below the value separating the second and third tertile of $z$ score in patients known to have coeliac disease referred from the gastrointestinal department. This was -0.5 at the femoral neck (FN) (nHanes III) and -1.1 at L2-4 (LS) (Hologic database), both values equating to a $T$ score of -2.5 at age $60-65$ years. Anti-endomyseal antibody testing was performed, and in 18 patients, anti-tTG titres (Celikay Varelisa; Phadia $\mathrm{GmbH}$ ) were also available. In addition, a full history was taken from all patients. Conditions associated with CD included thyroid disease, diabetes mellitus, epilepsy, inflammatory bowel disease, taking iron or B12 supplements, primary biliary cirrhosis, primary hyperparathyroidism, inclusion body myositis, renal calculi and Addison's disease: dermatitis herpetiformis was not included since it was assumed that patients had CD. Patients testing positive $(n=38)$ were compared with the same number of randomly selected patients testing negative (controls). Mean and SD are reported. Student's $t$ test was used for quantitative data, Fisher's exact test for categorical data and Pearson's $r$ test for correlation.

M. W. J. Davie $(\bowtie)$

Charles Salt Centre, Robert Jones and Agnes Hunt Hospital,

Oswestry, Shropshire SY10 7AG, UK

e-mail: mike.davie@rjah.nhs.uk
Patients and controls were of similar age ( 64.9 vs $64.5 ; p=$ ns). $Z$ scores in CD were lower at LS $(-1.84 \pm 1.31)$ and FN $(-1.28 \pm 0.68)$ compared with controls (LS $-1.14 \pm 1.05$ and FN $-0.91 \pm 0.45 ; p<0.01)$. There were 27 patients with 47 fractures in the serologically positive group and 32 patients with 49 fractures in the control group. However, serologically positive patients were more likely to have associated conditions (34 conditions in 22 patients) than patients testing negative (11 conditions in 7 patients) $(p<0.001)$. In serologically positive patients, $z$ score and age were positively correlated at LS $(r=0.43 ; p<0.01)$ only. Risk factors were equally distributed across the age range, and patients with risk factors had similar anti-tTG scores as those without.

In this selected population, the absence of conditions associated with $C D$ had a $66 \%$ negative predictive value and the presence of associated conditions a $76 \%$ positive predictive value. The use of coeliac serology in the DXA department appears to be useful only if associated conditions are present. These conditions have no age predilection, but $\mathrm{BMD} z$ score tends to be less adversely affected in older women. Whether, in the population studied, this tendency is related to the duration of $\mathrm{CD}$ or whether $\mathrm{CD}$ has a lesser effect on calcium absorption from an intestine already affected by age [2] and oestrogen deficiency [3] is uncertain.

\section{References}

1. Hjelle AM, Apalset E, Mielnik P, Bollerslev J, Lundin KE, Tell GS (2014) Celiac disease and risk of fracture in adults - a review. Osteoporos Int. doi:10.1007/s00198-014-2683-8

2. Avioli LV, McDonald JE, Lee SW (1965) The influence of age on the intestinal absorption of 47-Ca absorption in post-menopausal osteoporosis. J Clin Invest 44(12):1960-1967

3. Heaney RP, Recker RR, Stegman MR, Moy AJ (1989) Calcium absorption in women: relationships to calcium intake, estrogen status, and age. J Bone Miner Res 4(4):469-475, PubMed PMID: 2816496 